\title{
Treatment of Chronic Alcoholism: An Integrated Approach
}

\section{Hemangi Rajput*}

Integrative Health care Practitioner, Essence Natural Health Clinic, Canada

\begin{abstract}
Alternative medicine coupled with conventional and psychosocial therapies has been shown to be greatly effective in treating chronic alcoholism with positive treatment outcomes. Herbs like Kudzu, Tangerine Peel, Gentian and Bupleurum have been used efficaciously to treat chronic alcoholism and reduce liver toxicity. This article reviews the herbs Kudzu, Tangerine Peel, Gentian and Bupleurum with respect to their actions on the enzymes alcohol and acetaldehyde dehydrogenase. We further explore the genetic and pathophysiological basis of alcoholism while unraveling genetic polymorphisms in the genes involved in metabolic and effector action pathways that have an important bearing on why some individuals are addicted to alcohol, have severe withdrawal response and increased tendency to relapse. In this article we mainly discuss the role of alternative medicine, specifically in context with the above mentioned herbs for their role in inhibiting production of acetaldehyde dehydrogenase, suppressing craving, regulating blood glucose balance and reducing hepatotoxicity in chronic alcoholics.
\end{abstract}

Keywords: Acetaldehyde dehydrogenase; Alternative medicine; Bupleurum; Chronic alcoholism; Gentian; Integrated medicine; Kudzu; Tangerine Peel

\section{Background}

Alcoholism has been described as early as $1700 \mathrm{BC}$ in "The book of Anni" where Egyptians described excessive intoxication in humans. In $900 \mathrm{AD}$, distillation was discovered and the industrial revolution heralded the production and massive consumption of alcohol [1]. Ever since, we have seen the physical and emotional impact of chronic alcoholism on lives of the alcoholics, their families and our society that encompasses the gamut of liver dysfunction, malignancies, neurological impairment, employment issues and socio-legal problems.

Chronic alcoholism affects about 18 Percent of male population and 6 percent of female population in North America. Approximately 14 million Americans are addicted to alcohol with 88,000 deaths occurring every year due to excessive alcohol consumption [2]. Multiple or single organ failure from chronic alcoholic disorder is the cause of death in most of the cases. The underlying cause of death goes undermined and the risk factor for death arising from chronic alcoholism is often overlooked. Alcoholism is often multi-factorial involving physical, mental and social aspects; yet only the physical symptoms are treated primarily. Family history of alcoholism plays an important role in determination of an individual to be alcoholic; nevertheless triggers such as psychosocial impairment contributes to the existing disorder.

The physical symptoms due to cirrhosis of liver and/or end organ damage can come in the later stage of alcoholism. There are only behavioural changes that can be seen at the onset of alcoholic disorder. The initial stage of behavioural changes of alcoholism gets unnoticed by physicians until there is an evidence of physical damage. Women who are binge drinkers or chronic alcoholics have a devastating effect on the fetus during pregnancy, the fetus thus developing fetal alcoholic syndrome and has small for gestational age birth. Alcoholism as a disorder has genetic, environmental and behavioural attributes that requires an integrated multifaceted treatment approach to treat and prevent alcoholism in both chronic alcoholics and those with predisposition to alcoholism.

We need a health care model where integrated health care services work together to diagnose, treat and give information to alcoholics that would enable them to make informed choices about the available treatment options.
Alternative treatment with herbs has shown to be effective in ameliorating the side-effects of withdrawal, restore optimal homeostasis and decrease psychological dependence in chronic alcoholics.

\section{Pathophysiology of Chronic Alcoholism}

To treat alcoholism, we need to understand how ethanol is metabolized within the human body and the effects of excessive consumption. Ethanol consumed by humans is metabolized in the liver by enzyme alcohol dehydrogenase (ADH) to acetaldehyde. Acetaldehyde is a known carcinogen and is further oxidized to acetate by acetaldehyde dehydrogenase (ALDH). Acetate is then broken down into carbon dioxide and water for excretion. Rate of metabolism of alcohol is dependent on variations in the level and activity of $\mathrm{ADH}$ and ALDH enzymes. ALDH deficiency increases the levels of acetaldehyde formed in the body, leading to facial flushing, tachycardia and nausea. This limits the amount of alcohol that the individual can consume, and thus reduces the risk of chronic alcoholism in these subjects. On the other end of the spectrum, individuals with strong family history of chronic alcoholism have increased ALDH level that lets them metabolize large amounts of alcohol, and are thus prone to heavy or binge drinking, and are more likely to be chronic alcoholics [3].

Alcohol acts on the brain in a biphasic manner. When the blood alcohol levels are rising, it stimulates the brain by releasing norepinephrine in the brain, whereas the depressant effects are more pronounced when the alcohol levels are falling. Alcohol affects the neurons in the $\gamma$-aminobutyric acid (GABA) system and the endorphin system leading to the release of dopamine. Alcohol acts on the reward pathway of the brain composed of nucleus accumbens, the ventral tegmental area, and a part of the prefrontal cortex, and indirectly

${ }^{*}$ Corresponding author: Hemangi Rajput, Integrative Health care Practitioner Essence Natural Health Clinic, Canada, Tel:613-888-2559; E-mail: essenceclinic@yahoo.com

Received Janaury 31, 2014; Accepted February 21, 2014; Published February 24,2014

Citation: Rajput H (2014) Treatment of Chronic Alcoholism: An Integrated Approach. Altern Integ Med 3: 152. doi:10.4172/2327-5162.1000152

Copyright: (c) 2014 Rajput $\mathrm{H}$. This is an open-access article distributed under the terms of the Creative Commons Attribution License, which permits unrestricted use, distribution, and reproduction in any medium, provided the original author and source are credited. 
increases the dopamine activity which is responsible for the cravings seen in alcoholics [4]. The action of alcohol on GABA system induces relaxation and drowsiness, while its action on endorphin system is responsible for its pain-killer effect. By its action on glutamate system, it causes staggering, slurred speech and memory blackouts.

\section{Genetic Basis of Chronic Alcoholism}

Single nucleotide polymorphism (SNP) of the genes encoding the enzymes, alcohol dehydrogenase-1B $(A D H 1 B)$ and aldehyde dehydrogenase-2 (ALDH2) impact the production and/or oxidation of acetaldehyde [5]. Genetic polymorphisms in these genes affect the metabolism of alcohol in the body leading to variable amounts of acetaldehyde and reactive oxygen species generation [6].

Besides the enzymes involved in alcohol metabolism, several studies have looked at the imbalance between dopamine and serotonin levels in the brains of chronic alcoholics. The dopamine $\mathrm{D} 2$ receptor (DRD2) gene has been implicated in the genetic association studies looking at chronic alcoholism. Specifically, the DRD2 Taq1A A1 allele has been associated with decreased availability of dopamine D2 receptor and deficient binding capacity in the brain. Since alcohol increases dopamine activity, it may be used to compensate for this deficiency of dopamine activity [7]. Also, of note is the fact that serotonin deficiency in the brain results in alcohol-seeking behavior in humans and animals. The serotonin transporter gene (SLC6A4) has been implicated in alcohol association studies. The short risk allele of a polymorphism in the promoter region of SLC6A4 (5-HTTLPR) has been associated with decreased concentrations of serotonin transporter and less serotonin reuptake compared to the long allele [8]. Several studies have linked DRD2 and SLC6A4 risk alleles to alcohol abuse and psychological dependence. Meta-analyses have demonstrated DRD2 and SLC6A4 alleles associated with the risk of alcohol abuse and psychological dependence [9].

\section{Conventional Therapy for Chronic Alcoholism}

In the 1980 s it was discovered that $20-40 \%$ of East Asian origin subjects developed peripheral vasodilatation, facial flushing, tachycardia, headache, nausea and emesis on alcohol ingestion [10]. This was attributed to a point mutation in the gene coding for the high affinity mitochondrial $A L D H 2$ gene; which decreases the affinity for $\mathrm{NAD}^{+}$and makes the dehydrogenase enzyme virtually inactive. These findings explain the mechanism of action of disulfiram (Antabuse ${ }^{\mathrm{R}}$ ), a drug that non-specifically inhibits ALDH2, and so the patient develops all the above mentioned symptoms on alcohol ingestion leading to aversion for alcohol [11].

Dopamine plays an important role in alcohol addiction. Ethanol activates the mesolimbic dopamine system in humans and $\gamma$-aminobutyric $\operatorname{acid}_{A}\left(G_{A B A}\right)$ receptors are responsible for ethanol's reinforcing and several withdrawal-related effects via the mu-opiod receptors in the corticomesolimbic system. The opiod receptor antagonist, Naltrexone is a Food and Drug Administration approved drug that disrupts the cascade leading to striatal dopamine release following alcohol intake. It blocks opiod-peptide-mediated disinhibition of dopaminergic afferents in the midbrain to ventral striatum, and thus reduces craving and/or alcohol-induced stimulation [12].

\section{Alternative Medicine for Chronic Alcoholism}

Kudzu (Pueraria montana), also known as 'Japanese arrowroot' is a perennial vine found in parts of Asia and has been used for thousands for years in traditional Chinese medicine. The vine has roots that can grow to gigantic proportions and contains isoflavone glycosides like daidzin, daidzein and puerarin.

The structure of one of the key ingredients in Kudzu root, 7-O-glucosyl-4'-hydroxyisoflavone (daidzin) and ALDH2 was analyzed by Lowe et al. [13]. It showed the isoflavone moiety of daidzin binding near the aldehyde substrate-binding site in a hydrophobic cleft and the glucosyl function binding to a hydrophobic patch directly outside the isoflavone-binding pocket [13]. These findings explain the specificity and affinity of daidzin (IC50=80 nM) and the affinity of analogues with different substituent's at the glucosyl position [13].

Daidzin is a reversible selective inhibitor of ALDH2 that has been shown to suppress ethanol consumption in Syrian golden hamsters [14]. Reduced drinking due to ALDH2 inhibition is credited to aversive properties of acetaldehyde accumulated during alcohol intake.

A double-blinded clinical trial in human subjects was conducted at the McLean hospital to test the efficacy of Kudzu extract. Fourteen human volunteers with drinking problems were given either a placebo or $500 \mathrm{mg}$ capsule of Kudzu extract containing 19\% puerarin, $4 \%$ daidzin and $2 \%$ daidzein. The study demonstrated that 1 week of treatment with Kudzu extract resulted in a substantial reduction in the amount of alcohol consumption in a simulated natural environment. The data suggested that Kudzu prolonged or enhanced the acute effects of the first drink. The treated individuals decreased intake to a lower amount because the first few drinks satiated their desire for alcohol. This might be especially relevant in moderate to heavy drinkers who find it difficult to decrease their consumption during each drinking episode. There were no side effects reported with the Kudzu extract that would thwart long term compliance [15].

Kudzu extracts have been used for thousands of years in China as a hangover remedy. One of the common reasons for failed treatment and compulsive drug-seeking seen in chronic alcoholics is because of anxiety experienced after alcohol abstinence. NPI-031G (puerarin), an isoflavone isolated from Kudzu, is known to neutralize anxiogenic effects linked to alcohol. It has been shown that NPI-O31G (50 and 150 $\mathrm{mg} / \mathrm{kg}$ ip) significantly increased the social interaction and locomotor activity that have been reduced following abstinence from more than 17 days of alcohol (7\%) diet. The effects of NPI-031G are similar to those of the benzodiazepine antagonist, flumazenil $(5 \mathrm{mg} / \mathrm{kg})$, and the $5-\mathrm{HT}$ (2C) antagonist, SB $242084(1 \mathrm{mg} / \mathrm{kg})$. Considering the fact that longterm GABA agonists such as rimonabant, a cannabinoid CB1 receptor inhibitor, induce unwanted mood changes and suicidal tendencies; it has been proposed that GABA antagonistic properties may indeed be quite beneficial especially in long-term therapy and hence, the beneficial role of puerarin in the treatment of chronic alcoholism [16].

The other groups of Herbs that are widely used in treatment of alcoholism include the bitter herbs Gentian and Tangerine peel. They exert their effect through the recently characterized bitter taste receptors ( $\mathrm{TAS}_{2} \mathrm{Rs}$ ) involved in bitter substance-evoked gut responses at nanomolar concentrations up to 1 part in 12,000 [17]. These receptors have evolved in humans to avoid involuntary ingestion of toxic substances like TASR46 response to strychnine, an acetylcholine receptor antagonist that can cause convulsions and death [18]. Tangerine peel extracts are used for their role as metabolic enhancers, detoxifiers and in prevention of free radical damage. They have been shown to modulate transcription factors in the hippocampal neurons that may potentially be beneficial in preventing alcohol relapse due to its association with dopaminergic genetics, memory enhancement and good decision making abilities $[17,19]$. Bitters are also useful for 
their role in symptomatic relief of nausea, gastro-intestinal reflux, vomiting, abdominal pain, flatulence, constipation and loss of appetite [20]. Gentian is known for causing aversion to certain substances that can be harmful when ingested. A missense mutation in $T A S_{2} R 16$ gene decreases the receptor sensitivity bitter-taste stimulus which is associated with increased risk for being alcohol dependent [21].

A SNP within the $\operatorname{TAS}_{2} R_{9}$ receptor alters its ligand binding properties and normal functioning in the glucose and insulin homeostasis [22]. Gentian and Tangerine peel can prevent the effects of compromised $T A S_{2} R_{9}$ polymorphisms by their positive effect on glucose metabolism [23].

It has been shown that individuals with increased bitter taste perception, as defined by sensitivity to the bitter tasting chemical 6-n-propylthiouracil, have less frequent consumption of alcoholic beverages compared to their counterparts who are less sensitive to the compound; and a SNP located within the TAS R13 gene (rs1015443 [C1040T, Ser259Asn]) is associated with an increased alcohol intake [21]. Another SNP K172N, located in the $h T A S_{2} R 16$ gene that encodes a taste receptor for bitter beta-glucopyranosides, was shown to show a statistically significant association with alcohol dependence $(\mathrm{p}=0.00018)[24]$.

Finally, we review the drug Radix Bupleuri used in the treatment of chronic alcoholism. It is a perennial herb belonging to the genus Bupleurum. The name of the plant originated from the Latin word boupleuron (bous $=0 x$, pleutalon=ribs) describing the shape of the roots that are used for medicinal purposes [25]. Studies in rats with carbon tetrachloride induced liver damage had normalized liver functions with a daily dose of crude saponins of B. Falcatum [26]. Traditional Chinese medicine has been using this herb for its immunomodulatory, anti-inflammatory antiviral, anti-ulcer and anti-proliferative and hepatoprotective activities [27].

\section{Conclusion}

A recent pilot study involving ten heavy drinkers showed that when a blended formulation of the above described herbs viz. Kudzu, Gentian, Tangerine Peel and Bupleurum were administered, they showed statistically significant $(p=0.00298)$ decrease in the Alcohol Use Disorders Identification Test (AUDIT) scores [28]. Thus we suggest that the herbs Kudzu, Tangerine Peel, Gentian, and Bupleurum are very effective and should be used in combination of other therapies to achieve excellent treatment outcome in chronic alcoholism.

\section{References}

1. Hogarth W (1751) Gin lane, in Hogarth's Graphic Works, in Ronald Paulson Yale University Press.

2. Adams PE, Martinez ME, Vickerie JL, Kirzinger WK (2011) Summary health statistics for the U.S. population: National Health Interview Survey, 2010. Vital Health Stat 10: 1-117.

3. Cederbaum Al (2012) Alcohol metabolism. Clin Liver Dis 16: 667-685

4. Boileau I, Assaad JM, Pihl RO, Benkelfat C, Leyton M, et al. (2003) Alcohol promotes dopamine release in the human nucleus accumbens. Synapse 49 226-231.

5. Seitz HK, Stickel F (2007) Molecular mechanisms of alcohol-mediated carcinogenesis. Nat Rev Cancer 7: 599-612.

6. Gao CM, Takezaki T, Wu JZ, Zhang XM, Cao HX, et al. (2008) Polymorphisms of alcohol dehydrogenase 2 and aldehyde dehydrogenase 2 and colorectal cancer risk in Chinese males. World J Gastroenterol 14: 5078-5083.

7. Blum K, Sheridan PJ, Wood RC, Braverman ER, Chen TJ, et al. (1996) The D2 dopamine receptor gene as a determinant of reward deficiency syndrome. J R Soc Med 89: 396-400.
8. Hariri AR, Holmes A (2006) Genetics of emotional regulation: the role of the serotonin transporter in neural function. Trends Cogn Sci 10: 182-191.

9. Munafò MR, Matheson IJ, Flint J (2007) Association of the DRD2 gene Taq1A polymorphism and alcoholism: a meta-analysis of case-control studies and evidence of publication bias. Mol Psychiatry 12: 454-461.

10. Mizoi Y, Tatsuno Y, Adachi J, Kogame M, Fukunaga T, et al. (1983) Alcohol sensitivity related to polymorphism of alcohol-metabolizing enzymes in Japanese. Pharmacol Biochem Behav 18 Suppl 1: 127-133.

11. Chick J, Gough K, Falkowski W, Kershaw P, Hore B, et al. (1992) Disulfiram treatment of alcoholism. Br J Psychiatry 161: 84-89.

12. Anton RF, Drobes DJ, Voronin K, Durazo-Avizu R, Moak D (2004) Naltrexone effects on alcohol consumption in a clinical laboratory paradigm: temporal effects of drinking. Psychopharmacology (Berl) 173: 32-40.

13. Lowe ED, Gao GY, Johnson LN, Keung WM (2008) Structure of daidzin, a naturally occurring anti-alcohol-addiction agent, in complex with human mitochondrial aldehyde dehydrogenase. J Med Chem 51: 4482-4487.

14. Keung WM, Lazo O, Kunze L, Vallee BL (1995) Daidzin suppresses ethanol consumption by Syrian golden hamsters without blocking acetaldehyde metabolism. Proc Natl Acad Sci U S A 92: 8990-8993.

15. Lukas SE, Penetar D, Berko J, Vicens L, Palmer C, et al. (2005) An extract of the Chinese herbal root kudzu reduces alcohol drinking by heavy drinkers in a naturalistic setting. Alcohol Clin Exp Res 29: 756-762.

16. Overstreet DH, Kralic JE, Morrow AL, Ma ZZ, Zhang YW, et al. (2003) NPI-031G (puerarin) reduces anxiogenic effects of alcohol withdrawal or benzodiazepine inverse or 5-HT2C agonists. Pharmacol Biochem Behav 75: 619-625.

17. Kawahata I, Yoshida M, Sun W, Nakajima A, Lai Y, et al. (2013) Potent activity of nobiletin-rich Citrus reticulata peel extract to facilitate cAMP/PKA/ERK/ CREB signaling associated with learning and memory in cultured hippocampal neurons: identification of the substances responsible for the pharmacological action. J Neural Transm 120: 1397-409.

18. Matsubayashi H, Alkondon M, Pereira EF, Swanson KL, Albuquerque EX (1998) Strychnine: a potent competitive antagonist of alpha-bungarotoxinsensitive nicotinic acetylcholine receptors in rat hippocampal neurons. J Pharmacol Exp Ther 284: 904-913.

19. Bowirrat A, Chen TJ, Oscar-Berman M, Madigan M, Chen AL, et al. (2012) Neuropsychopharmacology and neurogenetic aspects of executive functioning: should reward gene polymorphisms constitute a diagnostic tool to identify individuals at risk for impaired judgment? Mol Neurobiol 45: 298-313.

20. Dotson CD, Shaw HL, Mitchell BD, Munger SD, Steinle NI (2010) Variation in the gene TAS2R38 is associated with the eating behavior disinhibition in Old Order Amish women. Appetite 54: 93-99.

21. Dotson CD, Wallace MR, Bartoshuk LM, Logan HL (2012) Variation in the gene TAS2R13 is associated with differences in alcohol consumption in patients with head and neck cancer. Chem Senses 37: 737-744

22. Dotson CD, Zhang L, Xu H, Shin YK, Vigues S, et al. (2008) Bitter taste receptors influence glucose homeostasis. PLoS One 3: e3974.

23. Yamada H (1995) [Structure and pharmacological activity of pectic polysaccharides from the roots of Bupleurum falcatum L]. Nihon Yakurigaku Zasshi 106: 229-237

24. Hinrichs AL, Wang JC, Bufe B, Kwon JM, Budde J, et al. (2006) Functional variant in a bitter-taste receptor (hTAS2R16) influences risk of alcohol dependence. Am J Hum Genet 78: 103-111.

25. Quattrocchi U (2000) CRC World Dictionary of Plant Names: Common Names, Scientific Names, Eponyms, Synonyms, and Etymology. Boca Raton, FL: CRC Press.

26. Abe $H$, Orita $M$, Konishi $H$, Arichi $S$, Odashima $S$ (1985) Effects of saikosaponin-d on enhanced $\mathrm{CCl}$-hepatotoxicity by phenobarbitone. J Pharm Pharmacol 37: 555-559.

27. Ashour ML, Wink M (2011) Genus Bupleurum: a review of its phytochemistry pharmacology and modes of action. J Pharm Pharmacol 63: 305-321.

28. Kushner S, Han D, Oscar-Berman M, William Downs B, Madigan MA, et al. (2013) Declinol, a Complex Containing Kudzu, Bitter Herbs (Gentian, Tangerine Peel) and Bupleurum, Significantly Reduced Alcohol Use Disorders Identification Test (AUDIT) Scores in Moderate to Heavy Drinkers: A Pilot Study. J Addict Res Ther 4: 153. 